\title{
Enhanced Side Information Generator with Accurate Evaluations in Block-Based Wyner-Ziv Video Coding
}

\author{
Chang-Ming Lee ${ }^{1,2}$, Jui-Chiu Chiang ${ }^{1,2}$, Zhi-Heng Chiang ${ }^{2}$, Kuan-Liang Chen ${ }^{1}$, \\ and Wen-Nung Lie ${ }^{1,2}$ \\ ${ }^{1}$ Department of Electrical Engineering \\ ${ }^{2}$ Department of Communication Engineering \\ National Chung Cheng University, Chia-Yi, 621, Taiwan, ROC \\ \{changminglee, rachel\}@ee.ccu.edu.tw, a1010820a2002@hotmail.com, \\ $\{\mathrm{klchen}$ samlab.ee, wnlie@ $\} \mathrm{ccu}$. edu.tw
}

\begin{abstract}
Wyner-Ziv coding (WZC) has received a lot of attention lately. Based on the block unit for WZ-/intra-coding and the temporal distance between two consecutive key blocks, two techniques are proposed to improve the performance of block-based distributed video coding. Depending on the spatiotemporal analysis, the first method adjusts the mode assignment to a more precise generation of side information. In addition, the second method carries out the correlation calculation in the encoder side to ensure a better statistical estimation between the side information frame and the original frame. Thus, the more accurate side information combined with the more reliable statistical parameters results in an improved coding efficiency. The simulation results show that the compound of these two proposed methods has an improvement up to $2.7 \mathrm{~dB}$ with respect to the previous work in block-based WZC.
\end{abstract}

Keywords: Wyner-Ziv coding, distributed video coding.

\section{Introduction}

Today's video coding standards, such as MPEG-X, H.26X, etc., are based on predictive coding techniques which use motion estimation (ME) to eliminate the temporal redundancy. The complexity of this kind of video codec is high due to ME process. These techniques are practical for applications where information is encoded only once and decoded many times. However, some applications require low complexity at the encoder side, and possibly disregard high complexity at the decoder side, such as wireless sensor networks. Slepian-Wolf theorem [1] and Wyner-Ziv (WZ) theorem [2] indicate a possibility to design coding schemes to fit the above requirements: The Slepian-Wolf theorem illustrates that given two correlated sources, the rate of independent and lossless encoding is greater than or equal to the rate of joint encoding, which means that the coding efficiency of the joint encoding technique is higher. On the other hand, the WZ theorem is a lossy version of the Slepian-Wolf theorem, exhibiting the rate region for encoding one source without any knowledge of the second source, and decoding this source with side information generated from the second one. 
When the above two algorithms are adopted in video coding, it is usually called distributed video coding (DVC). The pixel-domain DVC has been proposed in [3]. The conventional DVC architecture is based on a predefined group of picture (GOP). Frames in a video are organized as I-WZ-I-WZ..., where the GOP size is 2. Usually, I frames are recognized as key frames and are intra-coded, which are responsible for generating the side information for WZ frames at the decoder side. With more accurate side information, the bitrate required for $\mathrm{WZ}$ frames reconstruction in the decoder could be reduced and the coding efficiency will be better. Therefore, some researchers focused on the side information estimation improving [4-6].

Recently, due to the complicated implementation for WZ frames reconstruction, fast algorithms were proposed to speed up the process of WZ frames reconstruction. A parallelized DVC scheme [7] with an effective estimation is proposed to manage the decoding of subsequent bitplanes. Another issue in WZ video coding is the improvement of channel coding. Brites et al. [8] proposed a method to estimate the channel model parameter more accurately, which is then capable of providing a more reliable initial probability for turbo decoding. The channel model parameter is estimated at frame, block, or pixel level, for different granulations. Varodayan et al. [9] modified the LDPC codec such that it is rate-adaptive in contrast to the traditional fixed-length LDPC codec. Some researches considered parts of transmission as a virtual channel [10]. This virtual channel was analyzed and modeled. For example, they brought in the relation between the video compression ratio and the sensitivity of the estimated channel model parameter at the decoder side.

However, the temporal correlation between consecutive frames is not stationary and thus the fixed GOP structure will not be efficient enough. Therefore, a method to overcome the shortcoming of the traditional scheme was presented in [11] where the idea of a dynamic temporal distance (called temporal group of blocks (TGOBs)) between two consecutive key blocks was proposed. In attempt to improve the side information accuracy and also ease the design of the decoder, we propose two techniques in this paper where the key/WZ block decision rule is modified and the estimate of the noise model is performed in the encoder side rather than in the decoder side.

\section{Key-Block-Based Wyner-Ziv Coding}

In [12], the I-WZ-I-WZ ... coding structure is maintained, but some blocks in the WZ frames may be changed into intra-coding, meaning that the WZ frames may be encoded in mixed modes. On the other hand, blocks of the key frames are all intracoded. The gain of this coding-mode change for WZ frames is not high enough. On the other hand, Ascenso et al. [13] made the GOP size (i.e., the distance between two consecutive key frames) adjustable, according to the motion activity along the temporal domain within the sequence. However, these two techniques did not consider the tiny content variations in both the spatial and the temporal directions.

These issues motivate us to explore the spatial and temporal correlation in a finer granularity. In the spatial direction, a block is adopted as intra- or WZ encoding after block-mode decision. In the temporal direction, the GOP structure/length is dynamic, depending on the variations of temporal correlation in the video contents. 
Fig. 1 illustrates the key-block-based coding scheme based on the conventional pixel-domain DVC architecture [11]. At the encoder, the coding mode for each block is determined by the block-mode decision unit, which is then recorded in the blockmode map queue. If an intra mode is determined, the intra coding of H.264 is realized. Otherwise, the WZ coding (WZC) is performed. To be compatible to the conventional WZC process, before channel coding, a WZ frame generator manages the identified WZ blocks to form a full frame by replacing the key blocks with zeros. Similar to [4], the WZC consists of a uniform quantizer, a lower-density parity-check (LDPC) encoder, buffer, a feedback channel and a LDPC decoder.

At the decoder part, the intra bitstream is decoded to reconstruct the key blocks, which are then sent to the decoded key-block queue. Then, the decoded key blocks are used to generate the side information for WZ block by interpolating associated key blocks along the temporal direction. By integrating the reconstructed key and WZ blocks from both decoders, a complete frame is reconstructed.

For frame-based DVC schemes, the side information generated by interpolating two consecutive key frames is used to reconstruct the WZ frame by LDPC-decoding. If the side information is accurate enough, less parity bit are required for LDPC decoding and a better RD performance can be achieved.

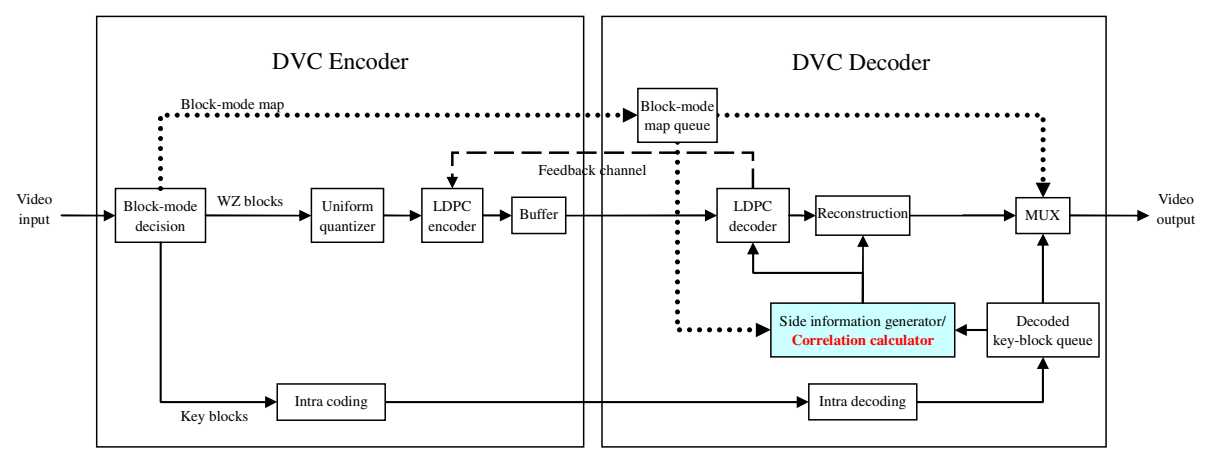

Fig. 1. Block-based Wyner-Ziv coding architecture

However, as mentioned above, the key-block architecture targets at providing a fine granularity in both the spatial and temporal directions, which may result in a compromise between an accurate estimation of the WZ information and an increase of the transmission bitrate. Let the structure of "IB-WZB...WZB-IB-WZB-..." (IB: intra-coded block, WZB: WZ-coded block) in the temporal direction be named temporal group of blocks (TGOBs). To achieve high coding efficiency, the TGOB size should be large for still background areas and small for dynamic foreground regions; it implies that TGOB size is dynamic and time-varying, according to the video contents.

\subsection{Block-Mode Decision Unit}

In our previous block-based DVC scheme, each frame is divided into non-overlapping blocks (size is 16 X 16 pixels). Each block is categorized into an IB or a WZB 
according to some criteria. All blocks in the first frame are intra-coded and serve as the initial key blocks along the sequence.

The block-mode is determined based on the temporal correlation with the previous key block at the co-located position and on the spatio correlation within a block. Two criteria in [12] are adopted:

$$
\begin{gathered}
S A D=\sum_{(x, y) \in \text { Block }_{i, j}}\left|B^{i, j, t}(x, y)-B^{i, j, t-d_{1}}(x, y)\right| \\
\sigma^{2}=\frac{1}{S} \sum_{(x, y) \in \text { Block }_{i, j}}\left|B^{i, j, t}(x, y)\right|^{2}-\left(\frac{1}{S} \sum_{(x, y) \in \text { Block }_{i, j}} B^{i, j, t}(x, y)\right)^{2}
\end{gathered}
$$

where $t$ is the time index, $(i, j)$ indicates the block index, $(x, y)$ is the pixel coordinate, $d_{1}$ is the distance to the previous key block (in the temporal direction), $S$ is the size of a block, and all $B$ 's represent the input video data. If $S A D$ is higher than a predetermined threshold or $\sigma$ is below the other given threshold, the block is identified as a key-block and will be intra-coded. Otherwise, it is WZ-coded. However, a suitable upper bound $U$ of TGOB size could be used to avoid a long decoding delay and inaccuracy accumulation. The block-mode map for each frame is recorded and transmitted to the decoder.

\subsection{Side Information Generator and Correlation Calculator}

Linear interpolation is used to estimate the WZ blocks from the decoded key-blocks. Let $d_{1}$ and $d_{1}$ be the temporal distances of the current WZ block to the preceding and successive key-blocks, respectively. Then pixels of a $\mathrm{WZ}$ block $W Z B^{i, j, t}$ are estimated by:

$$
W \hat{Z} B^{i, j, t}(x, y)=\frac{d_{2} \cdot I \hat{B}^{i, j, t-d_{1}}(x, y)+d_{1} \cdot I \hat{B}^{i, j, t+d_{2}}(x, y)}{d_{1}+d_{2}}
$$

where $I \hat{B}^{i, j, t}(x, y)$ represents the decoded key-block data. A collection of $\left\{W \hat{Z} B^{i, j, t}(x, y)\right\}$ form the side information in decoding the corresponding WZ block. Note that the side information also includes the block-mode map, which helps the system insert zero blocks at key-block positions (recall that the key-blocks are replaced with zeros to form a WZ frame).

In order to calculate the noise correlation in the block $W Z B^{i, j, t}$, the block variance $\sigma_{i, j, t}^{2}$ is obtained as the block-level correlation estimation mentioned in [8]. In conventional schemes, this statistical value is derived in the decoder by the side information generator/correlation calculator showed in Fig.1. In order to adapt the Laplacian distribution to the difference between the WZ and the side information at the block level, the parameter $\alpha_{i, j, t}$ can be estimated with the variance $\sigma_{i, j, t}^{2}$ by

$$
\alpha_{i, j, t}^{2}=\frac{2}{\sigma_{i, j, t}^{2}}
$$




\section{Detials of the Proposed Scheme}

\subsection{Block-Based Wyner-Ziv Coding with Modified TGOB Structure}

For the proposed scheme, the temporal distance TGOB between consecutive key blocks is determined by the block-mode-decision unit and is limited by an upper bound $U$. An intra-coding mode (key block) is determined when the current block has a low correlation with the prior key block (often, the motion between them is significant), or the temporal distance from the prior key block equals to the maximum delay $(U)$. However, interpolating two key blocks in distance will not guarantee a reliable estimation for WZ block. For the scenario in high quality requirement, more key blocks can support an accurate generation of side information. Therefore, the performance of reconstruction of WZ block would be enhanced.

For high-quality applications, we need to modify the rule of block mode decision mentioned in Section 2.1. The revised coding structure will be in a form of "IB-WZB...WZB-IB-IB-WZB... ZB-IB-IB-....". In this way, two consecutive and non-adjacent key blocks will have high correlation, and then more accurate side information and a higher PSNR can be ensured. To cope with coding efficiency at the same time, one more rule is introduced in the block-mode decision unit as follows.

1. If TGOB is less then $U$ : two intra blocks are inserted (the identified current block and its co-located block in the previous frame).

2. ELSE: only one intra block is inserted; it implies the co-located MB is identified as a key block.

For example, Fig. 2 shows that the TGOB size varies in the order of 3, 4 and 1, for the Block $_{0,1}$ with $U$ (the upper bound of TGOB size) equals 4 . After adapting the proposed block-mode decision rule, Fig. 3 presents the new block mode distribution.

In Fig.3, the block in red circle in the left side is decided as key block according to the first condition, while the one in the right side is unchanged and marked as WZ mode followed by one key block according to the second condition. Although more blocks are intra-coded, WZC is more efficient in precisely generating side information and therefore reducing the parity bits required to reconstruct the WZ frames. To implement this modified scheme, an additional frame delay at encoder side is demanded. However, we can achieve a higher PSNR with this modified scheme as indicated by experimental results.

\subsection{Correlation Calculator Relocation}

Normally, the side information generator among the DVC decoder in Fig.1 usually involves the correlation calculator and $\mathrm{MCI}$ (motion compensation interpolation for the frame-based WZC structure). In Fig.4, the module of the correlation calculator is realized in the encoder. The estimate of the noise model between the block $W Z B^{i, j, t}$ and the side information would be carried out by using the original WZ block and the estimated one by interpolating associated original key blocks. The calculation of the 


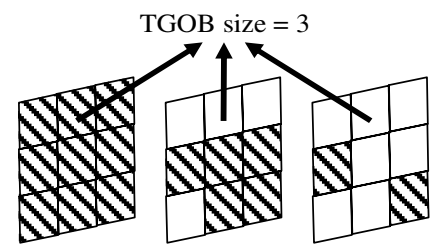

Frame-0

Frame-1

Frame-2

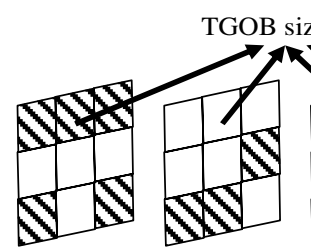

Frame-3

Fig. 2. The concept of dynamic TGOB with $U=4$, where the shaded blocks represent the key blocks and the others are WZ blocks

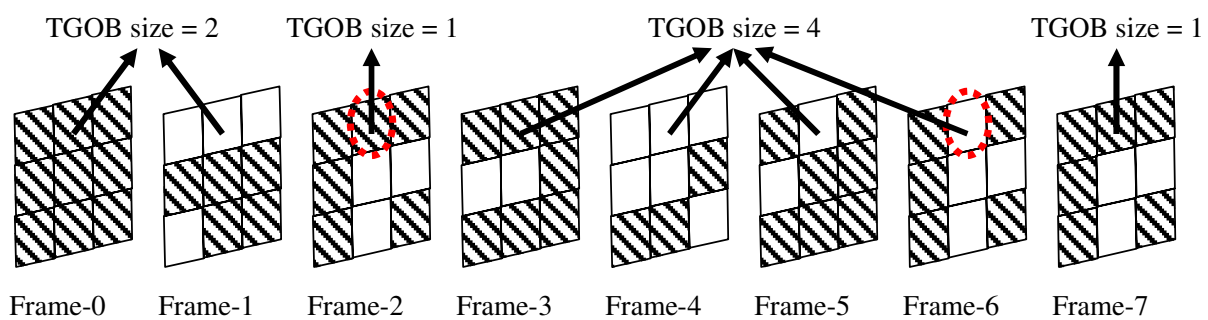

Fig. 3. The modified block-mode map according to highly correlated key blocks, with $U=4$

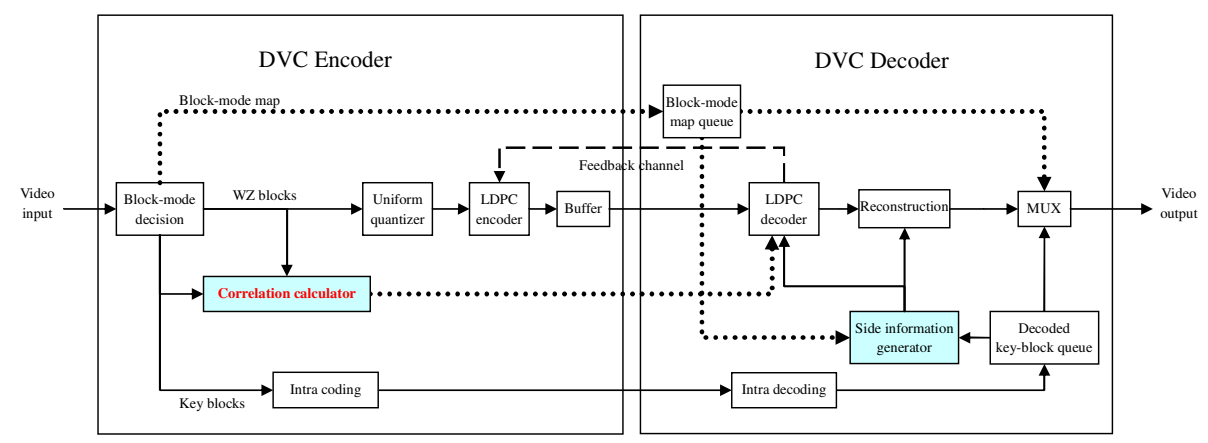

Fig. 4. Key-block-based Wyner-Ziv coding architecture with Correlation calculator in the DVC encoder

block variance $\sigma_{i, j, t}^{2}$ and the Laplacian distribution parameter $\alpha$ is similar to formulas in Section 2.2.

Consequently, this novel technique would cause a complexity shift from the decoder to the encoder. According to the block-based architecture, linear interpolation is used in the DVC, instead of MCI (motion compensation interpolation). Besides, the sample size for the corresponding analysis $(\alpha)$ is $16 \times 16$. Thus, the complexity to construct $W Z B^{i, j, t}$ and obtain the related information in DVC encoder is still light. In addition, a buffer is required to preserve the content of key blocks decided by the block-mode decision. 
This correlation calculator includes side information generator from the source data. Obviously, side information in the encoder is more precise compared with the one in the decoder. Consequently, the requested parity bits for the LDPC decoder via the feedback channel are less. These statistic results would be transmitted to the DVC decoder for LDPC decoding. Besides, the additional bitrate loading is negligible, even compared with the traditional video codec.

\section{Simulation Results}

In our proposed WZ coding scheme, the involved parameters include: number $(M)$ of bit-planes requested by the LDPC decoder, lower $(L)$ and upper $(U)$ bounds of the TGOB size, thresholds for $S A D$ in (1) and $\sigma$ in (2), and the quantization parameter (QP) for intra-coding. In our experiments for one bit-plane coding $(M=1)$, we set the minimum and maximum temporal coding delays be 1 and $10(L=1$ and $U=10)$, respectively. The test sequences are all of QCIF format with $30 \mathrm{~Hz}$ frame rate. The key blocks are encoded by intra-mode in H.264/AVC standard.

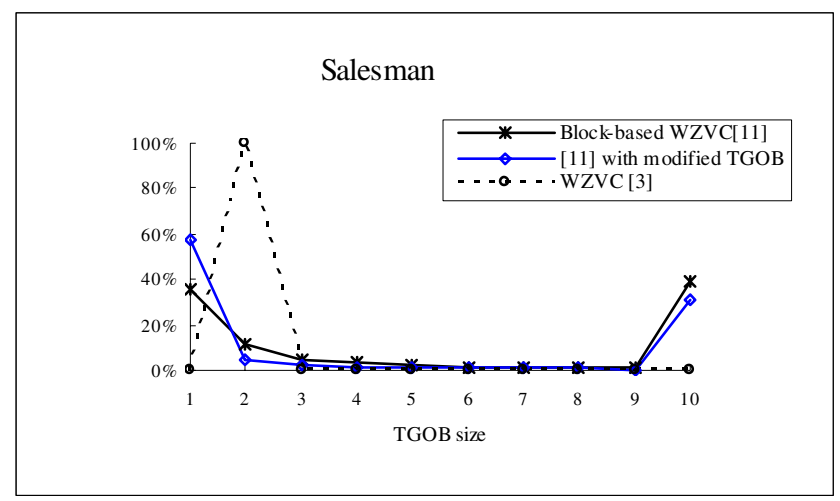

(a)

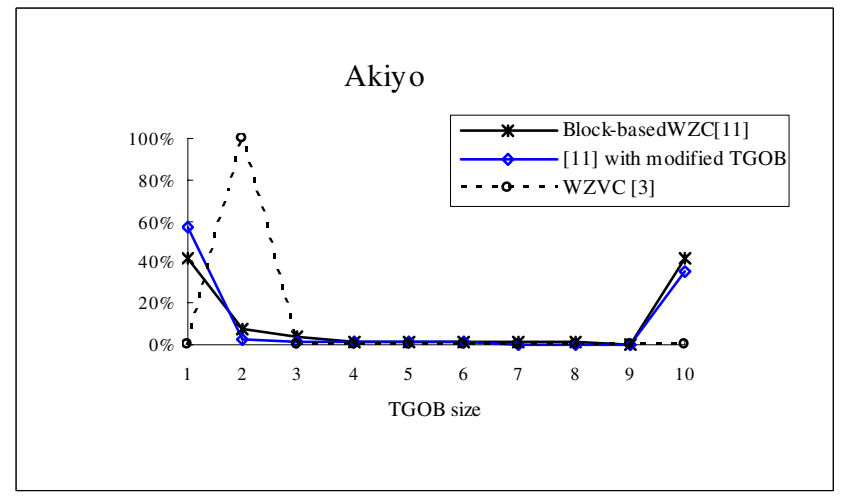

(b)

Fig. 5. The TGOB histogram for (a) Salesman sequence and (b) Akiyo sequence for the Blockbased WZVC and modified scheme with $U=10$ and conventional WZVC with GOP $=2$ 


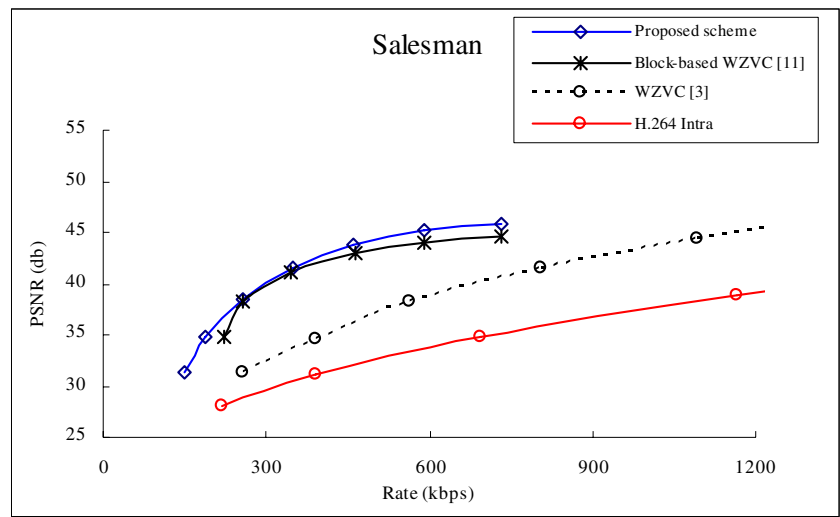

(a)

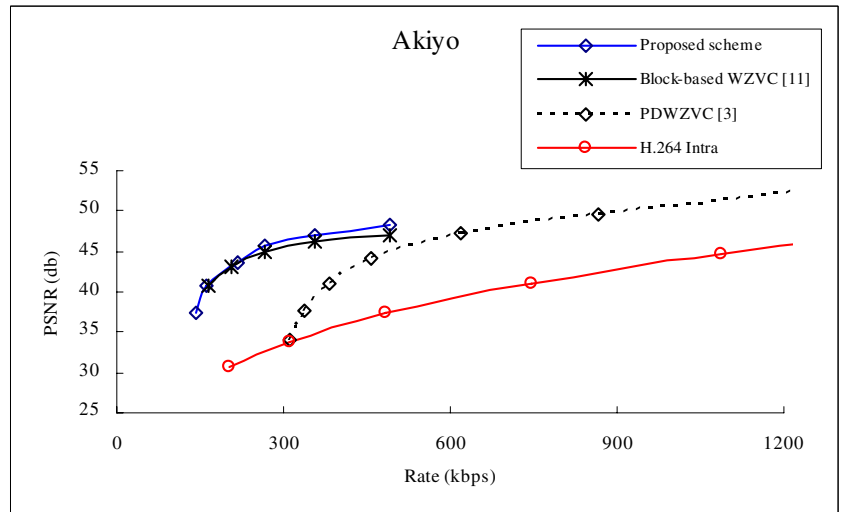

(b)

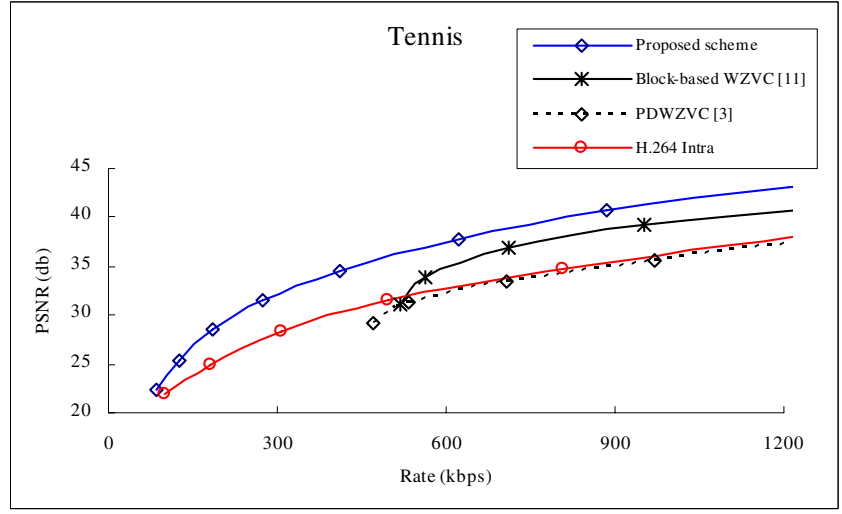

(c)

Fig. 6. RD performance for the sequences of (a) Salesman, (b) Akiyo and (c) Tennis 
The proposed codec is compared to two schemes in [3] and [11]. The first is a traditional LDPC-based DVC scheme adopting the frame-based "I-WZ-I-WZ..." coding structure (i.e., GOP size = 2) and a weighted-average interpolation, similar to (3), for side information generation. The other is block-based Wyner-Ziv coding, mentioned in Section 2.

Fig.5 shows the histogram of the TGOB size for sequences "Salesman" and "Akiyo" with $U=10$. For the conventional WZ codec [3], the GOP size is 2, which is similar to the coding with $\mathrm{TGOB}=2, L=2$ and $U=2$. This is not efficient for videos with stationary contents. A codec capable of adaptation to dynamic video contents will be desired. Ideally, a smaller TGOB size should be chosen for MBs with low temporal correlation, while a larger TGOB size is suitable for MBs with high temporal correlation. Compared to our previous work [11], there are $22 \%$ and $15 \%$ increase for TGOB = 1 in the process of "Salesman" and "Akiyo", respectively. This is due to the fact that we enforce some MBs to be intra-coded if they satisfy the conditions mentioned in Section 3.1.

Fig. 6 shows the rate-distortion performance of the proposed coding scheme where the bitrates and PSNRs are averaged over the whole sequence (291 frames). Fig. 6(b) reveals that the block-based WZC scheme in [11] has a PSNR gain up to $7.8 \mathrm{~dB}$ compared to [3] at the same bit-rate. This gain is even up to $11.8 \mathrm{~dB}$, compared to the H.264 intra coding $(\mathrm{GOP}=1)$. Moreover, the proposed new paradigm outperforms our previous work [11] and could achieve an improvement about $0.8,0.7$ and $2.7 \mathrm{~dB}$ in average for "Salesman", "Akiyo" and "Tennis", respectively. Besides, the proposed scheme also guarantees good performance in low quality/rate scenario. This achievement shows the proposed scheme has the ability to resist the instability of the channel coding.

\section{Conclusion}

In this paper, two approaches are proposed to increase the coding efficiency for block-based DVC scheme. First, more precise side information could be generated with an accurate evaluation by increasing the density of key blocks. Then, a more accurate noise model between the original WZ block and the associated side information is evaluated in the DVC encoder side. These two strategies aim at providing the LDPC decoder with more accurate information and less parity bit are required consequently. In particular, for the high bitrate, the improvement is obvious due to more key blocks. Furthermore, the correlation calculator in the encoder can still ensure satisfactory performance for the scenario of low bitrate. It reveals that the proposed DVC system is flexible in numerous applications.

\section{Acknowledgement}

This paper was supported by National Science Council of Taiwan (NSC 96-2221-E194-013-MY2). 


\section{References}

1. Slepian, D., Wolf, J.K.: Noiseless coding of correlated information sources. IEEE Trans. on Information Theory 19(4), 471-480 (1973)

2. Wyner, D., Ziv, J.: The rate-distortion function for source coding with side information at the decoder. IEEE Trans. on Information Theory 22, 1-10 (1976)

3. Aaron, A., Zhang, R., Girod, B.: Wyner-Ziv Coding of Motion Video. In: 36th Asilomar Conference on Signals, Systems and Computer, Pacific Grove, USA (November 2002)

4. Ascenso, J., Brites, C., Pereira, F.: Motion Compensated Refinement for Low Complexity Pixel Based Distributed Video Coding. In: IEEE Int'l Conf. on Advanced Video and Signal-Based Surveillance, Como, Italy (September 2005)

5. Kubasov, D., Guillemot, C.: Mesh-based motion compensated interpolation for side information extraction in distributed video coding. In: IEEE Int'l Conf. on Image Processing (ICIP), Atlanta, USA, October 8-11 (2006)

6. Ascenso, J., Brites, C., Pereira, F.: Improving frame interpolation with spatial motion smoothing for pixel domain distributed video coding. In: 5th EURASIP Conference on Speech and Image Processing, Multimedia Communications and Services, Slovak Republic, June 29 - July 2 (2005)

7. Tonomura, Y., Nakachi, T., Fujii, T.: Efficient index assignment by improved bit probability estimation for parallel processing of distributed video coding. In: IEEE Int'l Conf. on Acoustics, Speech and Signal Processing (ICASSP), USA, March 31 - April 4 (2008)

8. Brites, C., Ascenso, J., Pereira, F.: Studying temporal correlation noise modeling for pixel based Wyner-Ziv video coding. In: Proc. of IEEE Int'l Conf. on Image Processing (ICIP), Atlanta, USA, October 8-11 (2006)

9. Varodayan, D., Aaron, A., Girod, B.: Rate-adaptive codes for distributed source coding. EURASIP Signal Processing 86, 3123-3130 (2006)

10. Westerlaken, R.P., Borchert, S., Gunnewiek, R.K., Lagendijk, R.L.: Dependency channel modelling for a LDPC-based Wyner-Ziv video compression scheme. In: Proc. of IEEE Int'1 Conf. on Image Processing (ICIP), Atlanta, USA (October 2006)

11. Tsai, D.-C., Lee, C.-M., Lie, W.-N.: Dynamic key block decision with spatio-temporal analysis for Wyner-Ziv video coding. In: IEEE Int'l Conf. on Image Processing (ICIP), USA, September 16 - October 19 (2007)

12. Tagliasacchi, M., Trapanese, A., Tubaro, S., Ascenso, J., Brites, C., Pereira, F.: Intra mode decision based on spatio-temporal cues in pixel domain Wyner-Ziv video coding. In: IEEE Int'1 Conf. on Acoustics, Speech, and Signal Processing (ICASSP), Toulouse, France, May 14-19 (2006)

13. Ascenso, J., Brites, C., Pereira, F.: Content adaptive Wyner-Ziv video coding driven by motion activity. In: IEEE Int'l Conf. on Image Processing (ICIP), USA, October 8-11 (2006) 